\title{
Identification of glycoproteins secreted by wild-type Botrytis cinerea and by protein O-mannosyltransferase mutants
}

\author{
Mario González, Nélida Brito and Celedonio González*
}

\begin{abstract}
Background: Botrytis cinerea secretes a high number of proteins that are predicted to have numerous O-glycosylation sites, frequently grouped in highly O-glycosylated regions, and analysis of mutants affected in O-glycosylation has shown, in B. cinerea and in other phytopathogenic fungi, that this process is important for fungal biology and virulence.

Results: We report here the purification of glycoproteins from the culture medium, for a wild-type strain of $B$. cinerea and for three mutants affected in the first step of O-glycosylation, and the identification of components in the purified protein samples. Overall, 158 proteins were identified belonging to a wide diversity of protein families, which possess Ser/Thr-rich regions (presumably highly O-glycosylated) twice as frequently as the whole secretome. Surprisingly, proteins predicted to be highly O-glycosylated tend to be more abundant in the secretomes of the mutants affected in O-glycosylation than in the wild type, possibly because a correct glycosylation of these proteins helps keep them in the cell wall or extracellular matrix. Overexpression of three proteins predicted to be O-glycosylated in various degrees allowed to confirm the presence of mannose a1-2 and/or a1-3 bonds, but no mannose a1-6 bonds, and resulted in an enhanced activity of the culture medium to elicit plant defenses.
\end{abstract}

Conclusions: Glycosylation of secretory proteins is very prevalent in $B$. cinerea and affects members of diverse protein families. O-glycosylated proteins play a role in the elicitation of plant defenses.

Keywords: Botyris cinerea, Glyco-secretome, Mannosyltransferase

\section{Background}

Botrytris cinerea has been considered the second most important plant pathogenic fungus according to its economic and scientific importance [1], and is able to infect more than 200 plant species including many with high agronomic value [2]. The genome of two $B$. cinerea strains have been sequenced $[3,4]$ and one of its key features is the prediction of a high number of secretory proteins, about $10 \%$ of the polypeptides coded by the genome. Proteomic studies have actually revealed an abundant and diverse set of proteins in the extracellular medium for $B$. cinerea cultures [5-11]. This set contains mainly proteins involved in the degradation of plant structures (such as cellulases, xylanases, proteases, etc.),

\footnotetext{
*Correspondence: cglez@ull.es

U.D. Bioquímica y Biología Molecular, Universidad de La Laguna, 38206 La Laguna (Tenerife), Spain
}

but also proteins with other functions such as the induction of cell death in the host [12-14], as well as plenty of proteins with unknown function [7]. The role of some of these extracellular proteins has been studied by gene knock-out to identify secretome members contributing to virulence, i.e. virulence factors, but only a few have shown to contribute modestly to the infection process [14-19].

It is known that $O$-glycosylation is crucial in determining the structure and function of numerous secreted and membrane-bound proteins [20]. Carbohydrate chains have been proposed to enhance the stability and solubility of proteins, to confer protection against proteases, to act as a sorting determinant, and to be involved in various development and differentiation processes [20]. We have recently shown, by an in silico approach, that about half of predicted proteins in fungal secretomes display Ser/Thrrich regions, i.e. regions with at least $40 \% \mathrm{Ser} / \mathrm{Thr}$ in a 
minimum of 20 contiguous residues [21], which are usually considered to display high-density of $O$-glycosylation [21-23]. Indeed, about one fourth of secretory proteins were predicted to display hyper- $O$-glycosylated regions, i.e. regions with at least $25 \%$ of residues predicted to be $O$-glycosylated [21]. In fungi, $O$-glycosylation begins with the addition of a mannose residue by protein $\mathrm{O}$-mannosyltransferases (PMTs) in the lumen side of the endoplasmic reticulum (ER) membrane [24], a process which has recently been shown to occur, at least partially, concomitantly with the translocation of nascent polypeptides into the ER [25]. Three PMT families exist in fungi (PMT1, PMT2 and PMT4) [26,27], and filamentous fungi usually have only one pmt gene per family. The deletion of one or more of the pmt genes usually results in loss of viability or strong defects such as reduced conidia production, changes in fungal morphology, etc., emphasizing the importance of $\mathrm{O}$-glycosylation for the fungal biology. The B. cinerea genome also contains three pmt genes [28], bcpmt1, 2 and 4, and the three of them, but especially bcpmt2, are critical for the stability of the cell wall, are necessary for sporulation, and are required for the generation of the extracellular matrix. Besides, BcPMTs are also required for full virulence in a variety of hosts, with a special role in the adhesion to, and penetration of, intact plant leaves [28].

Since PMTs have been found in both prokaryotes and eukaryotes [24], but not in plants, these proteins are promising targets in the design of novel control strategies against fungal phytopathogens. However, the ERassociated topology of these proteins [29] poses strong problems for the elucidation of their structure and the design of specific inhibitors. An indirect approach is the identification of specific PMT substrates that could also be important for fungal biology and virulence. Since we have previously observed significant changes in the patterns of protein secretion and glycosylation by the bcpmt mutants [28], we have addressed here the characterization of glyco-secretome differences between wild-type $B$. cinerea and bcpmt mutants.

\section{Results}

Many proteins in the $B$. cinerea secretome are glycosylated

We have previously identified more than one hundred proteins in the B. cinerea secretome [7], and we have also observed that the appearance of the secretome in 1D- and 2D-PAGE changes radically for mutants affected in PMTs, which catalyse the first step of $O$-glycosylation [28]. This prompted us to better characterize the set of glycoproteins secreted by wild-type $B$. cinerea strain B05.10, as well as by the mutants lacking each one of the three $B$. cinerea PMTs [28]. Preliminary observations allowed us to establish static liquid cultures in Petri dishes with YGG-low medium, inoculated with mycelial plugs and incubated for 4 days, as the optimum conditions to maximize isolation of secretory proteins in the case of $\Delta b c p m t$ mutants. Culture media obtained from these plates contained plenty of proteins (Figure 1A) and the band pattern in SDS-PAGE was different for the wild type and for the $\Delta b c p m t$ mutants. Purification of glycoproteins from the culture medium by affinity chromatography with Concanavalin-A resulted in quite different band patterns (Figure 1B), with some proteins clearly disappearing and others being enriched. Notably, the band corresponding to the most abundant protein in the secretome of $B$. cinerea, the $35-\mathrm{kDa}$ aspartic protease BcAp8 [30] (black arrow in Figure 1A), is completely absent after purification of glycoproteins, in accordance with the fact that no $N$ - or $O$-glycosylated sites are predicted in silico for this
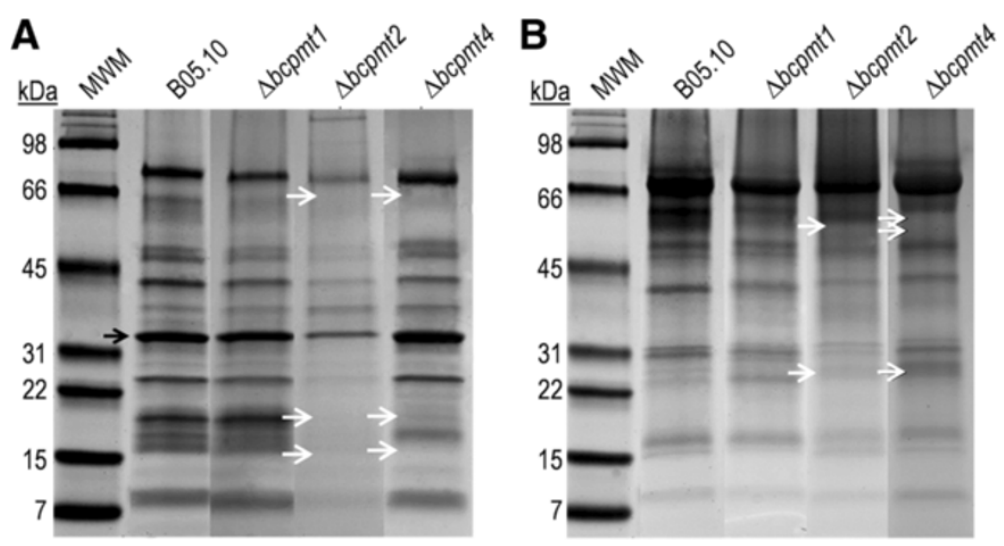

Figure 1 Glyco-secretome purification. Electrophoretic analysis of glycoproteins purified from the extracellular fraction in cultures of wild-type B. cinerea (B05.10) and the indicated $\Delta b c p m t$ mutants. A: SDS-PAGE showing all proteins precipitated from $150 \mu$ l of culture medium. B: SDS-PAGE of the purified glycoprotein samples $(150 \mu$ l). Black arrow: BcAp8, a protein with no predicted glycosylation sites. White arrows: example bands appearing/disappearing in the mutant samples as compared with the corresponding wild-type sample. 
protein by the NetNGlyc 1.0 [31] and NetOGlyc 4.0 $[32,33]$ servers. Moreover, an enrichment in proteins with high molecular weight $(>50 \mathrm{kDa})$ was observed for all samples after glycoprotein purification, in agreement with the fact that lectin-blot experiments also show that glycosylation is more prominent for these proteins [28]. As expected, the SDS-PAGE band pattern of purified glycoprotein samples is different for the wild type and the three $\Delta b c p m t$ mutants, especially in the case of $\Delta b c p m t 2$ and $\Delta$ bcpmt4.

2D electrophoresis of purified glycoproteins was carried out for B05.10 and the $\Delta b c p m t 1$ mutant, and resulted in a relatively simple spot pattern (Figure 2), with not too many differences for the two samples. Some spots, however, showed clear differences in intensity, such as spot 18 , which is over-expressed in the $\Delta b c p m t 1$

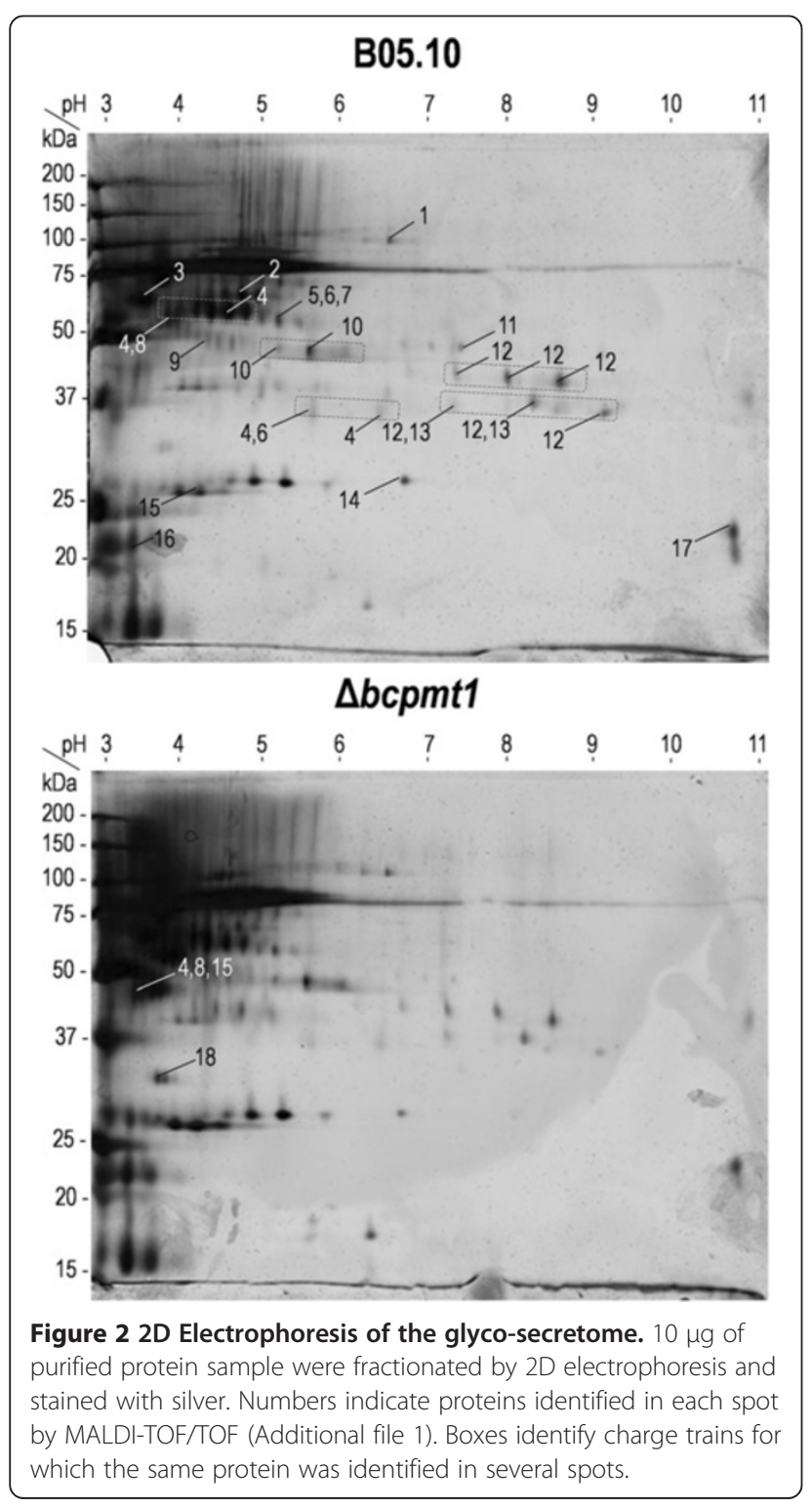

sample. A total of 29 spots obtained from the wild-type or the $\Delta b c p m t 12 \mathrm{D}$ gels were excised and analyzed by MALDI-TOF/TOF, resulting in the identification of 18 proteins (Additional file 1). In some cases (spots 4, 10, 12 and 13), the same protein was identified in several spots forming charge trains (different pIs), or even in spots (spots 4 and 12) with different mobility in the second dimension (different apparent molecular weight).

\section{Identification of glycoproteins in the secretome by LC-MS/MS}

The purified glycoprotein samples obtained for the four strains were also analyzed by shotgun proteomics. The total number of proteins identified was 157 in the four samples (Additional file 1). Only one of the 18 proteins identified previously from the $2 \mathrm{D}$ electrophoresis gels (spot 13, Pectin methylesterase BcPME1) was not identified by LC-MS/MS, so the total number of glycoproteins identified by the two methods is 158 . Most proteins identified (133 of 158) showed signal peptide according to SignalP $4.1[34,35]$, and 22 others showed alternative secretion features according to SecretomeP $2.0[36,37]$. Forty-eight of the 158 proteins are described here for the first time as components of the B. cinerea secretome, while the rest have been reported previously [5-11].

Contrary to our initial expectations, the composition of the glyco-secretome was not radically different for the wild type and for the three $\Delta b c p m t$ mutants, so that proteins clearly absent in one or more of the mutants, and therefore putative substrates of the corresponding BcPMT, are scarce. Restricting the comparative analysis to highly expressed proteins (Table 1), so that the number of spectral counts accumulated for each protein makes the comparison more significant, it results that the 13 proteins considered are all expressed in the three $\Delta b c p m t$ mutants, as well as in the wild type. Only two proteins, pectin methylesterase BcPME2 and B0510_6786 (of unknown function), have an abundance of less than $25 \%$ that of the wild type in at least one of the mutants. Noticeably, some of these proteins are actually found at elevated levels in the mutants, in comparison with the wild type. Overall, these results confirm a prominent role for PMTs in protein glycosylation, but in a way that may be more complex than anticipated. It does not seem, for example, that individual proteins are substrates of only one BcPMTs.

The 158 proteins were classified into families previously defined [7] (Additional file 1). Family assignments were made using sequence similarities with proteins of known function detected by BLAST [38], and the presence of conserved domains according to Pfam [39,40]. Distribution of glycoproteins in families was similar for the wild type and the three mutants (Figure 3). The glyco-secretome is composed mainly of polysaccharide hydrolases $(\sim 36 \%)$, followed by proteases $(\sim 14 \%)$ and oxidoreductases $(\sim 13 \%)$. The rest of the families $(\sim 37 \%)$ 
Table 1 Abundant proteins identified by LC-MS/MS in wild-type $B$. cinerea and the three $\Delta b c p m t$ mutants

\begin{tabular}{|c|c|c|c|c|}
\hline \multirow[t]{2}{*}{ Protein name ${ }^{1}$ - Gene ID } & \multicolumn{4}{|c|}{ Spectral counts $^{2}$} \\
\hline & B05 & $\Delta b c p m t 1$ & $\Delta b c p m t 2$ & $\Delta b c p m t 4$ \\
\hline 1,3-beta-glucanosyltransferase Glycolipid anchored surface protein - B0510_3559 & 16 & 22 & 21 & 25 \\
\hline Alpha-amilase (GH13) - BC1G_02623.1 & 13 & 12 & 5 & 23 \\
\hline Beta-1,3-endoglucanase with a GPI anchor (GH17) - B0510_9551 & 5 & 15 & 6 & 17 \\
\hline Glucoamylase (CBM20, GH15) - B0510_2137 & 137 & 152 & 52 & 201 \\
\hline Glucoamylase (CBM20, GH15) - B0510_2884 & 30 & 39 & 15 & 34 \\
\hline Oxidorreductase - B0510_547 & 9 & 20 & 15 & 13 \\
\hline Pectin methylesterase BCPME2 (CE8) - B0510_344 & 31 & 37 & 9 & 4 \\
\hline Phospholipase C - B0510_184 & 11 & 17 & 14 & 15 \\
\hline Protease (Merops A1) - B0510_6952 & 7 & 18 & 8 & 12 \\
\hline Pro-Xaa carboxypeptidase (Merops S28) - BC1G_09564.1/BC1G_09565.1 & 16 & 23 & 7 & 15 \\
\hline Unknown, similar to phytase - B0510_5392 & 12 & 17 & 14 & 20 \\
\hline Unknown, similar to subtilase family protein - B0510_6786 & 21 & 22 & 4 & 14 \\
\hline Unknown, similar to yeast spore wall proteins - BC1G_10630.1 & 8 & 12 & 6 & 15 \\
\hline
\end{tabular}

'Protein family and/or conserved domains found in sequences are included between brackets where possible: glycosyl hydrolase family (GH...), Merops family (Merops...), carbohydrate esterase family (CE...), or carbohydrate binding modules (CBM...).

${ }^{2}$ Only proteins fulfilling the following two conditions are displayed: they have at least 15 spectral counts in one of the four strains, and they account for more than $2 \%$ of all spectral counts in one of the strains.

are represented by a similar amount of proteins and contribute always less than $10 \%$ to the overall composition. As compared with the complete secretome [7], the glycosecretome showed an increase in polysaccharide hydrolases and oxidoreductases, but a reduction in pectinases.
Ser/Thr-rich and highly O-glycosylated regions in glycoproteins

We have recently analyzed in silico the presence and distribution of Ser/Thr-rich regions, as well as hyper$O$-glycosylated regions, in the predicted $B$. cinerea

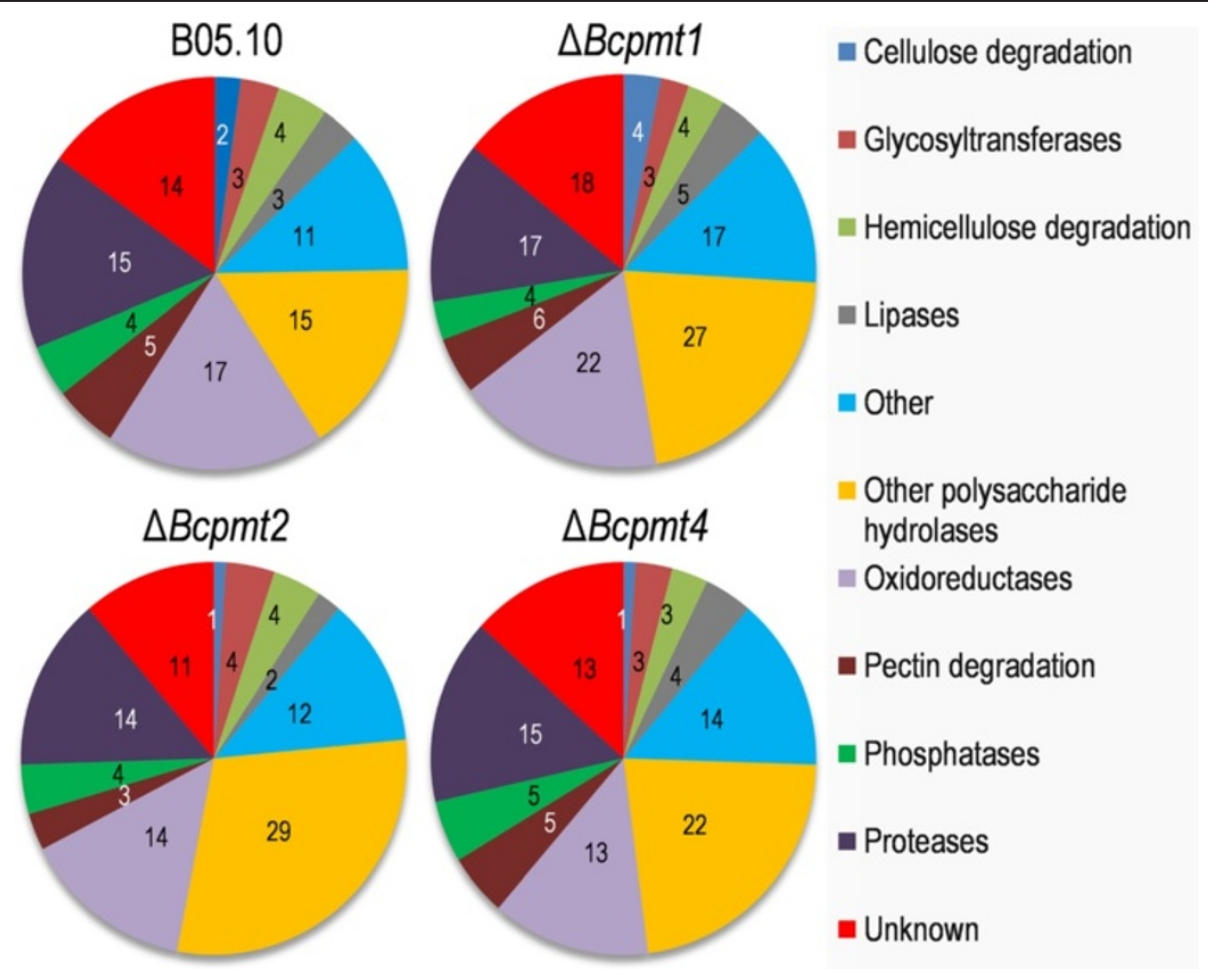

Figure 3 Family distribution of the glycoproteins identified in the four $\boldsymbol{B}$. cinerea strains. Numbers indicate the number of proteins in each category. 
secretome [21]. We now have the opportunity to compare those results with a similar analysis for the experimental set of actually secreted and glycosylated proteins reported here (the 158 proteins displayed in Additional file 1). The results show, in first place, that Ser and Thr residues are extremely abundant among secreted glycoproteins. On average, $38 \%$ of residues in these proteins are either Ser or Thr in the experimental glycoprotein set, while this number is just $17 \%$ for the whole predicted secretome. When the MS Excel XRR macro described previously [21] was used to study if these residues are grouped forming Ser/ Thr-rich regions (Figure 4A) a big number of these was found, even when a very high Ser/Thr content was necessary for a region to be considered as Ser/Thr-rich (up to $80 \%)$. The proportion of proteins displaying Ser/Thr-rich regions was considerably higher for the experimental set of glycoproteins than for the predicted whole secretome (Figure 4A). For example, 80\% of glycoproteins displayed regions with at least $40 \% \mathrm{Ser} / \mathrm{Thr}$, while only $46 \%$ of proteins in the whole predicted secretome exhibited them (Figure 4A). Moreover, the total number of Ser/Thr-rich regions was comparatively higher for the glycoprotein set: among the 158 glycoproteins 299 Ser/Thr-rich regions (with at least $40 \% \mathrm{Ser} / \mathrm{Thr}$ ) were found, almost 2 per protein, while the whole predicted secretome (1910 proteins) contained only 1501 of these Ser/Thr-rich regions. Notably, seven proteins were found with regions of 20-32 residues in which at least $80 \%$ of them are Ser or Thr.

Similar results were obtained when glycosylation was predicted for the set of purified glycoproteins. Both
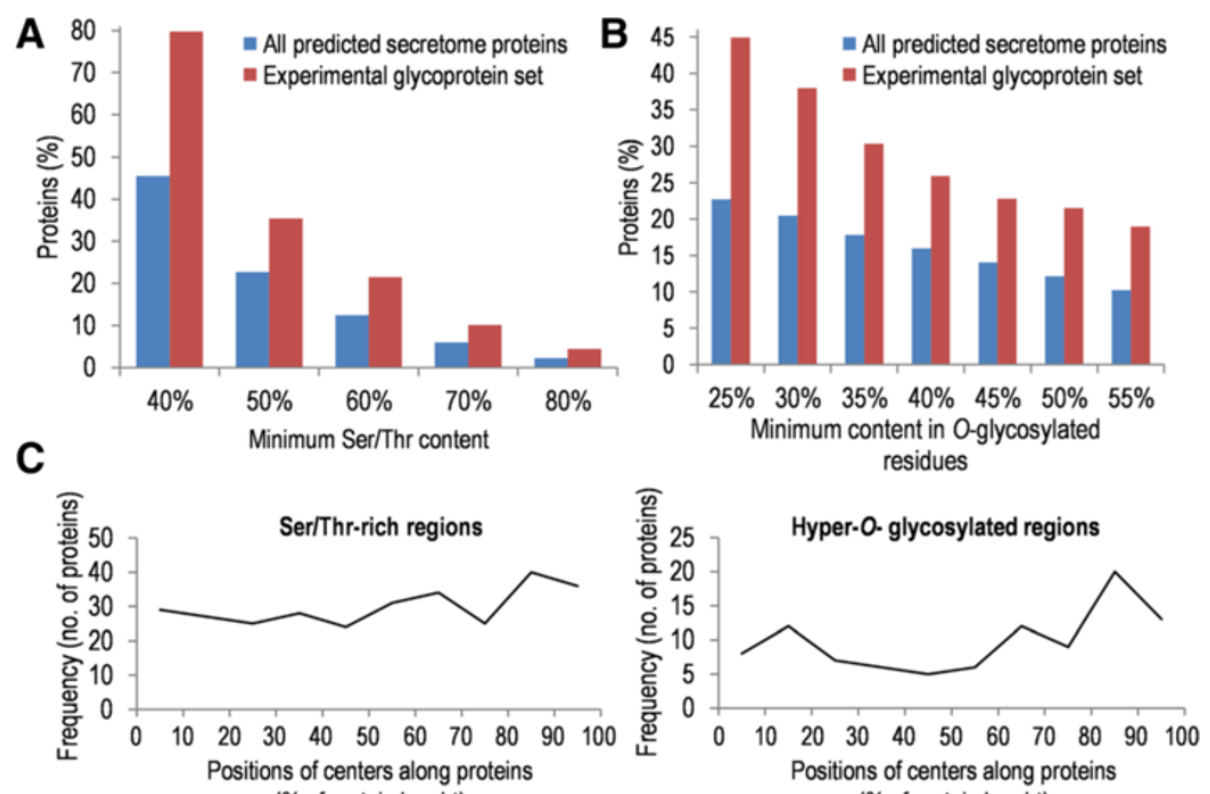

(\% of protein lenght)

(\% of protein lenght)

D

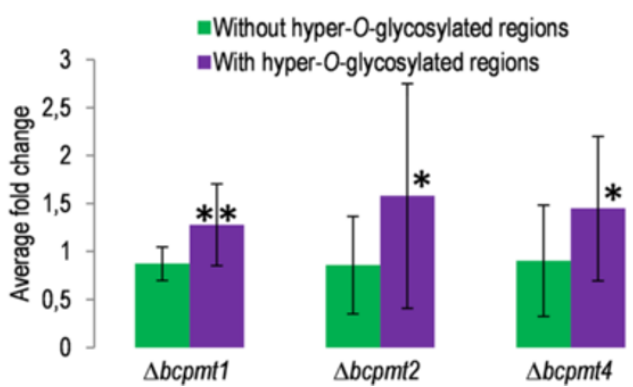

Figure $4 \mathrm{Ser}$ /Thr-rich regions and hyper-O-glycosylated regions among proteins in the glyco-secretome. A: Percentages of proteins displaying regions with the indicated minimal Ser/Thr content (horizontal axis), both among the experimental glycoprotein set (red bars) and among all predicted secretome proteins (blue bars). B: Percentage of proteins with predicted hyper-O-glycosylated regions in the same two proteins sets, also analyzed for the indicated minimal abundances of predicted O-glycosylation sites (horizontal axis). C: Frequency distribution of Ser/Thr-rich and hyper-O-glycosylated regions along proteins for the experimental glycoprotein set. The total number of regions found in every possible $10 \%$ interval along protein length is represented. $\mathbf{D}$ : Relative abundance of proteins in the $\Delta b c p m t$ mutants, as compared with the wild-type strain B05.10, estimated from the spectral counts for two protein sets: those displaying hyper-O-glycosylated regions (purple bars) and those without them (green bars). Asterisks indicate a significant difference for the two sets (t-test) at either $0.95\left({ }^{*}\right)$ or $0.99\left({ }^{* *}\right)$ confidence level. 
$N$-glycosylation and $O$-glycosylation was predicted for most of them ( $91 \%$ and $87 \%$ of proteins, respectively), but the average number of predicted $O$-glycosylated residues in proteins was about three times higher than the number of $N$-glycosylated ones. The predicted $O$-glycosylation positions were frequently grouped in highly-O-glycosylated regions (Figure $4 \mathrm{~B}$ ), but not the $N$-glycosylation sites (not shown). Using the less stringent definition of highly-Oglycosylated regions (regions of at least 20 residues with $25 \%$ or more $O$-glycosylation sites, see ref. 21 ), it results that about half of proteins (45\%) in the glycosecretome display these kind of regions, about twice the number obtained for the whole predicted secretome (23\%) [21].

We observed previously, for the whole predicted secretome, that Ser/Thr-rich and predicted highly O-glycosylated regions have a tendency to be located toward the two ends of polypeptide chains [21]. This tendency is clearer in the case of glycoproteins and especially evident for predicted highly O-glycosylated regions (Figure $4 \mathrm{C}$ ), which are frequently found in the $\mathrm{C}$-terminal region of proteins and are also common at the $\mathrm{N}$-terminus right after the signal peptide.

Interestingly, we found a relationship between the presence of predicted hyper-O-glycosylated regions in proteins and their relative abundance in the secretomes of the $\Delta b c p m t$ mutants, estimated from the spectral counts. This was done by first choosing a set of abundant proteins, for which we could calculate more reliable fold changes from spectral counts, consisting of 29 proteins with at least 10 spectral counts in one of the four Botrytis strains and a relative abundance of at least $1 \%$ in one of the four strains. The fold change in the amount of protein in the $\Delta b c p m t$ mutants, relative to the wild type, was then estimated from the spectral counts for each individual protein. Next, the average fold change was calculated separately for those proteins with predicted hyper-O-glycosylated regions and for those without them, and are displayed in Figure 4D. Statistically significant differences were found for the two groups in the three mutants, so that the relative abundance of proteins predicted to have hyper- $O$-glycosylated regions is, on average, 1.43 times higher in the secretomes of the $\Delta b c p m t$ mutants than in the secretome of the wild type. On the contrary, no difference was observed for the proteins without predicted hyper- $O$-glycosylated regions.

One possible explanation for the fact that proteins displaying hyper-O-glycosylated regions tend to be found at higher levels in the secretomes of the $\Delta$ bcpmt mutants is that $O$-glycosylation causes retention of the proteins in the cell wall or the extracellular matrix. These proteins, being glycosylated to a less extent in the $\Delta b c p m t \mathrm{mu}-$ tants, would escape easier and accumulate to higher levels in the culture medium. To test this hypothesis, two fusion proteins were expressed in B. cinerea which both contained the sequence of BcSpl1 [14], an abundant component of the $B$. cinerea secretome partially retained in the cell wall [41], fused to GFP, but differed in the presence of a Ser/Thr-rich region in the C-terminal end originally coming from the endoglucanase Cel5A [42]. Unfortunately, the level of expression seemed quite different for the two fusion proteins, as judged from the green fluorescence in the mycelium (Figure 5A) and from the western-blots with monoclonal anti-GFP antibodies (Figure 5B), and both gave various bands in the western-blot. By considering all the bands obtained in the blots, we determined for liquid cultures of each of these two strains the ratio of protein secreted to the culture medium versus the amount associated with the mycelium (Figure 5C). Although the effect of the introduction of the Ser/Thr-rich tail in the protein was not spectacular, the fusion protein displaying it showed a higher tendency to be associated with the mycelium which was statistically significant in the three repetitions of the experiment shown in Figure 5C.

\section{Overexpression of $\mathbf{O}$-glycosylated proteins in $\mathbf{B}$. cinerea}

Three of the proteins detected as components of the glyco-secretome were overexpressed in $B$. cinerea to further study them. These proteins were selected because they display Ser/Thr regions and are glycosylated in different degrees (Additional file 1). The three proteins were the endopolygalacturonase BcPG1, previously reported to be required for full virulence in $B$. cinerea [17] and to be perceived as a PAMP (Pathogen Associated Molecular Pattern) by Arabidopsis [43], and two proteins of unknown function that we have named BcIEB1 (B0510_2361, similar to IgE Binding proteins) and BcSUN1 (B0510_8844, similar to members of the yeast SUN family). These proteins differed in the number or length of the Ser/ Thr-rich regions, with BcSUN1 being predicted to be highly glycosylated by NetOGlyc 4.0 and the other two poorly glycosylated (Additional file 1).

Expression of the three genes under the control of the strong promoter oliC resulted in the accumulation of decent amounts of proteins in the culture medium (Figure 6). In order to check if, and how, these proteins were glycosylated we treated them with two glycosyl hydrolases: exo $\alpha 1-2,3$ and exo $\alpha 1-6$ mannosidases. Since all attempts to purify them from the culture media were unsuccessful, the treatment was done with a whole secretome sample and analyzed by western-blot with anti-c-myc antibodies (Figure 6). Reduction of protein size, as a consequence of treatment, was considerable for BcSUN1 (estimated in 20-25 kDa), limited for BcIEB1 (estimated in 1-2 kDa), and not detectable for BcPG1, in good accordance with the number of glycosylation sites predicted for the three proteins: 74, 3 and 1 (Additional file 1). This reduction was seen only for the incubation with the exo $\alpha 1-2,3$ 


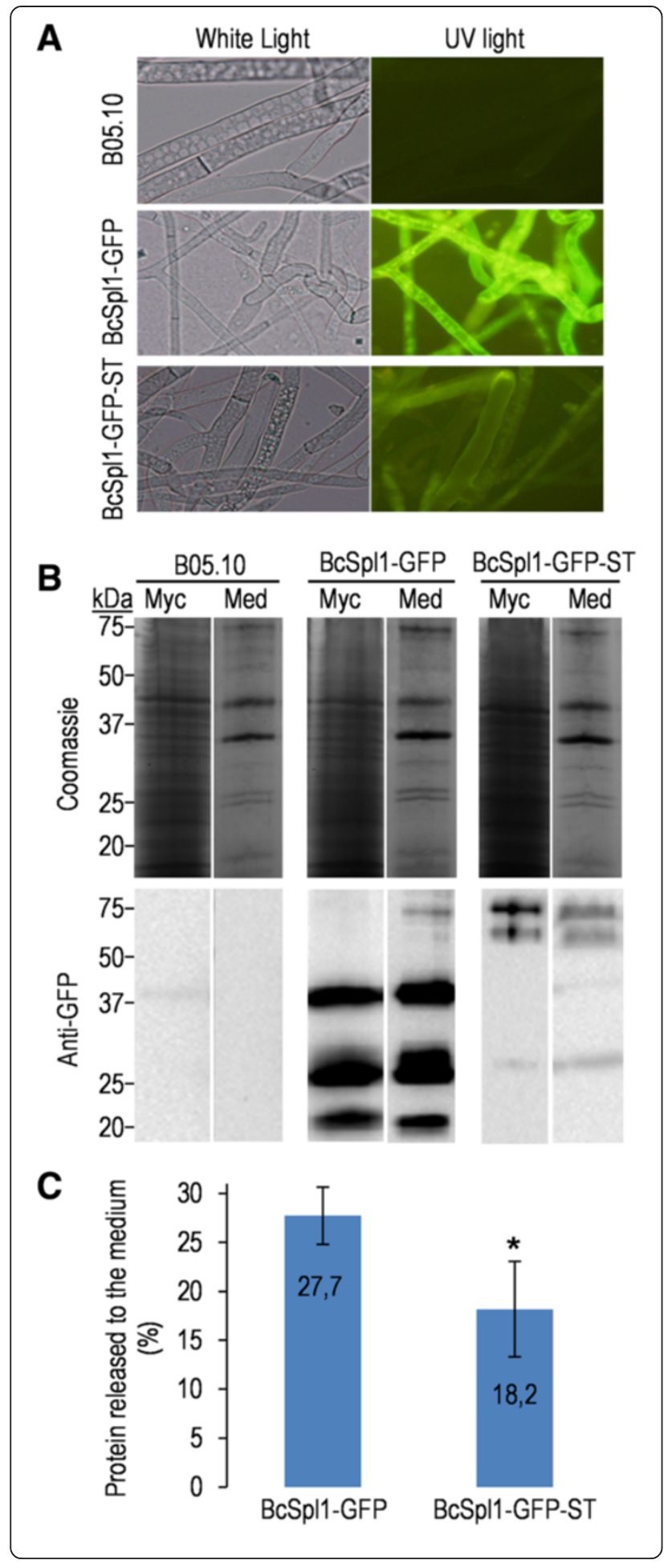

Figure $5 \mathrm{Ser} / \mathrm{Thr}$-rich regions partially retain proteins with the mycelium. A: Fluorescence of transformants expressing a fusion proteins with BCSpl1, GFP, and with (BCSpl1-GFP-ST), or without (BCSpl1-GFP), a Ser/Thr-rich region from Cel5A. The wild-type strain B05.10 was used as control. B: SDS-PAGE and western blot (anti-GFP) comparing the amount of recombinant protein released to the culture medium (Med) and the amount associated with the mycelium (Myc) in cultures (16 hours in YGG-low medium) of the same strains as in (A). Amounts of proteins loaded were those contained in $1.125 \mathrm{ml}$ of medium (1/67 of total in culture) or associated with $7.5 \mathrm{mg}$ of mycelium (1/122 of total in culture). C: Percentages of recombinant protein released to the medium as soluble protein for the two strains, averaged for three independent cultures. Asterisk indicates a statistically significant difference between the two strains with 0.95 confidence (Mann-Whitney test).

enzyme. Treatment with exo $\alpha 1-6$ mannosidase did not produced any effect by itself nor did it potentiate the effect of exo $\alpha 1-2,3$. These results indicate, therefore, that at least BcSUN1 and BcIEB1 are actually glycosylated, that the amount of sugars incorporated is in accordance with the predictions carried out by NetOGlyc, and that glycosidic chains contain primordially mannoses linked by $\alpha 1-2$ or $\alpha 1-3$ glycosidic bonds. Additionally, the same strategy was used to assess the incorporation of mannose residues to the BcSpl1-GFP-ST fusion protein described above (Figure 6), and also in this case treatment with exo $\alpha 1-2,3$ mannosidase (but not $\alpha 1-6$ ) produced a considerable reduction in size, but not in the case of the control fusion protein BcSpl1-GFP.

Culture media from the three strains overexpressing the $O$-glycosylated proteins were also tested for the ability to elicit defense responses in plants by seedling growth inhibition assay [44], a sensitive and quantitative test which correlates with typical plant defence responses such as callose deposition, production of reactive oxygen species, or pathogenesis-related gene expression [45,46]. In this assay, culture media from the three overexpressing strains revealed considerably more efficient in inhibiting growth of tobacco seedling, as compared with the media obtained with the wild-type strain (Figure 7A-C). Moreover, the seedlings treated with media from the overexpressing strains showed necrotic symptoms that were clearly more intense than with the wild-type strain.

\section{Discussion}

$O$-glycosylated proteins are crucial components of fungal secretomes. Not only has it been predicted that more than half of secretome components display $O$-glycosylation sites [21], frequently in the form of highly-O-glycosylated regions, but it has also been shown that mutants affected in the first step of the $O$-glycosylation machinery display a vast arrays of defects including decreased virulence in the case of phytopathogens $[28,47]$. By purification and identification of glycoproteins, we have shown here 

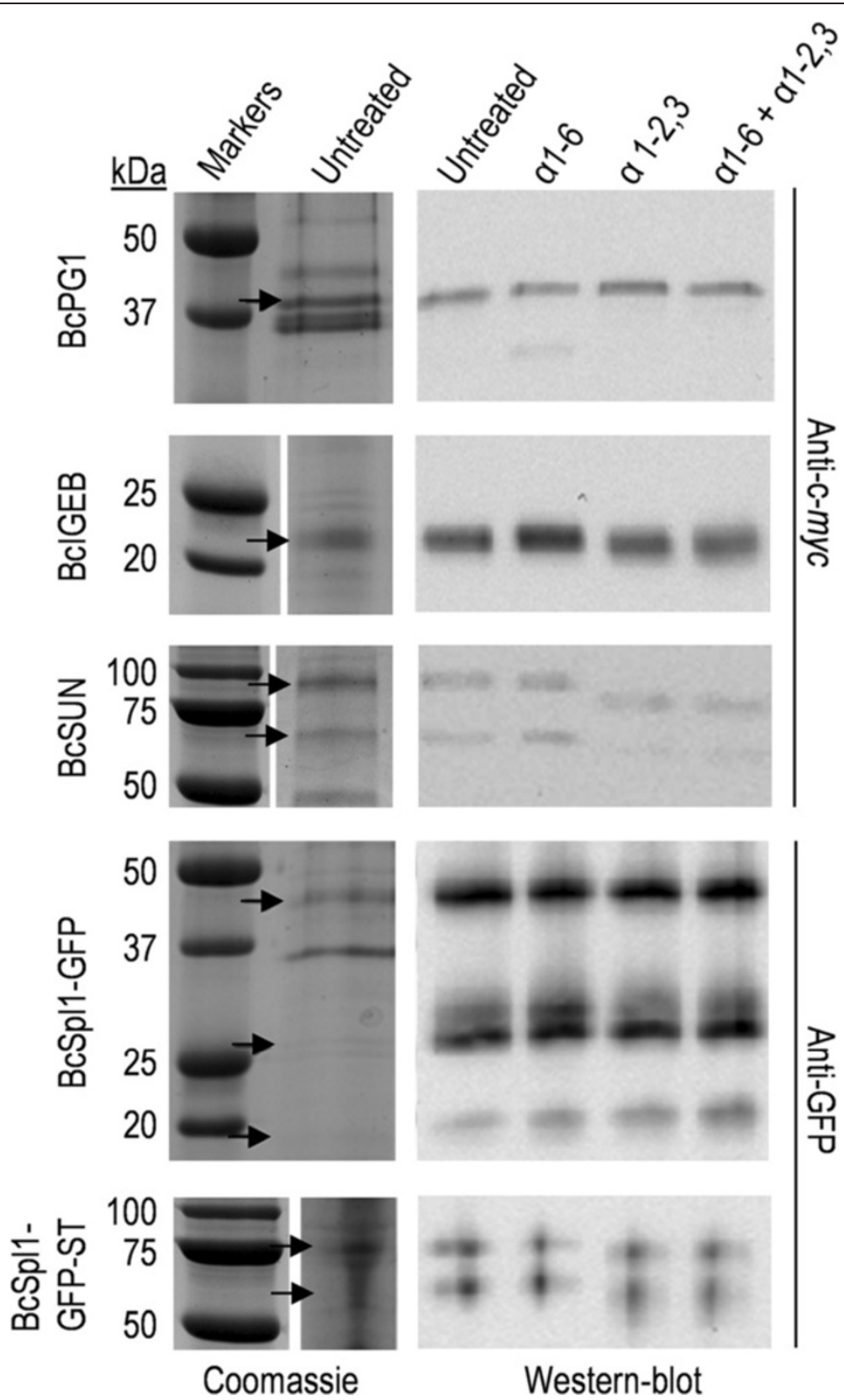

Figure 6 Deglycosylation of $B$. cinerea proteins with exo a1-2,3 and exo a1-6 mannosidases. Extracellular media from cultures of the strains overexpressing the indicated proteins were treated with the enzymes and analysed by western-blot with the indicated antibodies. SDS-PAGE of the untreated samples is also shown (Coomassie). The samples treated with the different enzymes, as well as the untreated controls, were identical and corresponded to $900 \mu \mathrm{l}$ of culture medium. Arrows point to the positions in the Coomassie-stained gels that correspond to the bands observed in the western blots.

that $B$. cinerea indeed secretes a substantial amount of these proteins. Altogether, 93 proteins were identified in the isolated glycoprotein sample for the wild-type strain B05.10, which include 18 proteins for which experimental evidence is provided here, for the first time, as components of the secretome. It is worth noting the absence, among these proteins, of the aspartic protease BcAP8, whose band is not detected in SDS-PAGE of the purified glycoprotein sample (Figure 1) nor does it appear among the 157 proteins identified by LC-MS/ MS for the same sample. This protein serves as an internal negative control for the purification of glycoproteins, since it is the most abundant protein in the secretome [7] but it lacks predicted glycosylation sites. Its absence in the purified sample indicates that the purification is working properly. Family distribution of 


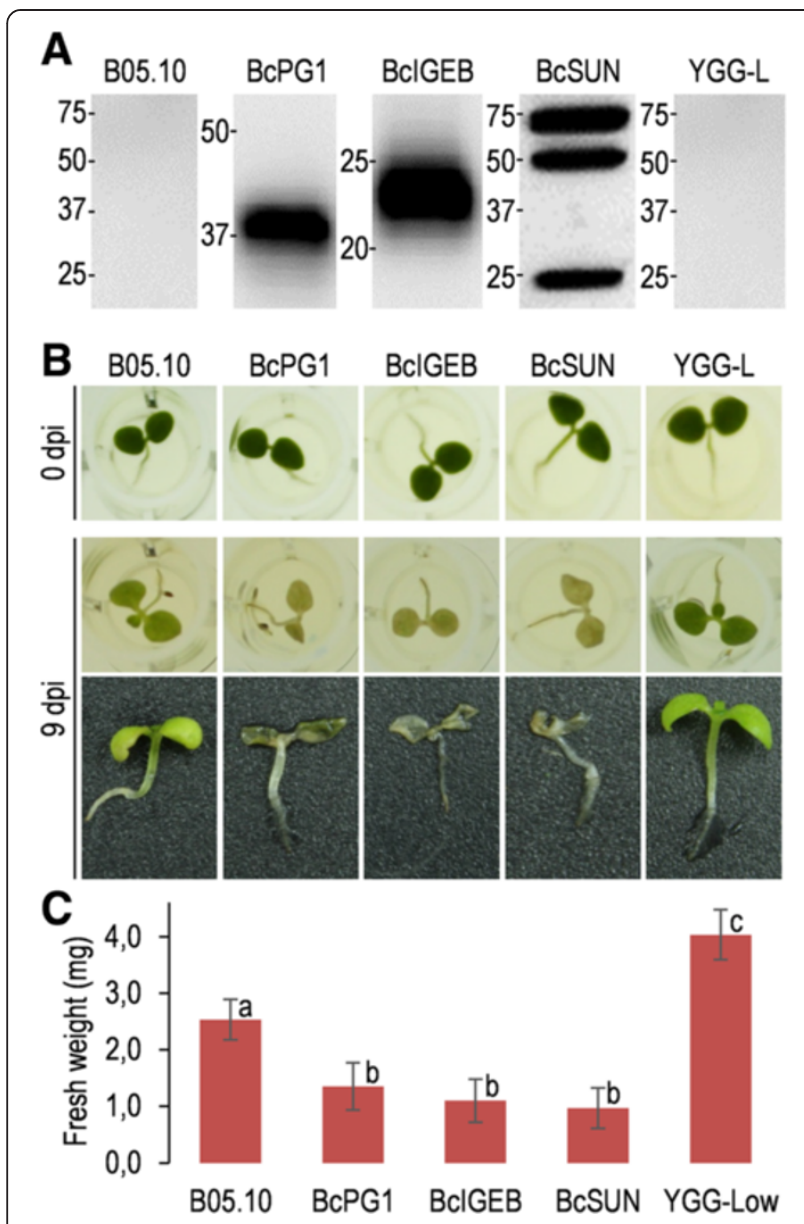

Figure 7 Growth inhibition and necrosis of seedlings caused by O-glycosylated proteins. Tobacco seedlings were treated for 9 days with culture media from the strains overexpressing the indicated O-glycosylated proteins and then assessed for necrosis and growth inhibition. A: Western-blot (anti-c-myc) showing the relative amounts of recombinant proteins in the culture media from the overexpressing strains. Medium from the wild-type strain (B05.10) and uninoculated medium (YGG-low) were used as controls. Each lane contained proteins precipitated from $1 \mathrm{ml}$ of medium. Molecular weight makers are show to the left of each lane $(\mathrm{kDa})$. B: Example seedlings treated with the culture media. C: Average weight $(n=6)$ of treated seedling after the 9-day incubation. Different letters on bars indicate statistically significant differences with 0.99 confidence.

glycoproteins (Figure 3) is not very different from that obtained for the whole secretome sample [7], with a low number of proteins classes containing most of the proteins: mainly proteases, polysaccharide hydrolases, oxidoreductases, and plenty of proteins with unknown functions. It does not seem, therefore, that glycosylation acts preferentially on specific types of proteins.

Contrary to what we expected, the composition of the glyco-secretome was not very different for the wild type and the three $\Delta b c p m t$ mutants affected in O-glycosylation. We reasoned that lack of glycosylation of specific proteins in one or more of the $\Delta b c p m t$ mutants would prevent its retention by the lectin affinity column and so would result in its absence in the corresponding glycoprotein sample. Although there are plenty of proteins (62\%) which are absent in one or more of the four strains, these correspond almost always to those with just one or a few spectral counts, so that their absence/ presence may be the result of mere chance. The comparison of spectral counts for those proteins with the highest expression (Table 1), however, does not show any protein which is absent in the mutant samples. This may indicate, at least for these proteins, that they are not substrates of single PMTs (or PMT dimers) but are glycosylated by the combined action of several of them. PMTs have been shown to act as specific dimers in Saccharomyces cerevisiae, Pmt1/Pmt2 heterodimer and Pmt4/Pmt4 homodimer [48], and as almost all possible hetero- and homo-dimeric combinations in Aspergillus nidulans [49]. Our results agree better with the situation in A. nidulans, since a more promiscuous association of PMT monomers, to form functional dimers, would inherently imply an easier substitution of one of the PMTs by the other isoforms.

Surprisingly, more often than not, spectral counts for the most abundant proteins are higher in the $\Delta b c p m t$ mutants than in the wild-type strain B05.10 (Table 1), possibly because proteins correctly glycosylated are retained easier in the cell surroundings (cell wall and/or extracellular matrix) while those with an incomplete glycan structure escape easier. It was actually shown (Figure 4D) that proteins predicted to have hyper-O-glycosylated regions are, on average, more abundant in the glycosecretomes of the $\Delta b c p m t$ mutants, as compared with the wild type, while the same is not true for the proteins not predicted to have hyper- $O$-glycosylated regions. Moreover, addition of a Ser/Thr rich region to a fusion protein expressed in $B$. cinerea increases the amount of it that remains associated with the mycelium (Figure 5), as compared with the amount found in the extracellular medium, although these results need to be taken with caution because of the different levels of GFP expression in the two strains. If confirmed, this effect of glycosylation maybe physiologically important for $B$. cinerea, as enzymes acting on soluble substrates and producing assimilable nutrients for the fungal cells may be more efficient if retained closer to the cells.

The overexpression of three of the proteins predicted to be $O$-glycosylated allowed experimental confirmation of the post-translation modification for two of them, as a decrease in the apparent molecular mass observed in SDS-PAGE was caused by enzymatic deglycosylation (Figure 6). Although prediction of $\mathrm{O}$-glycosylation carried out by NetOGlyc is only approximate for fungal proteins [21], the changes in the apparent molecular mass obtained for the three proteins are in accordance 
with the number of $O$-glycosylation sites predicted $(74,3$, and 1 for BcSUN1, BcIEB1, BcPG1, respectively). Incubation with two different deglycosylation enzymes showed the presence of mannose $\alpha 1-2$ and/or $\alpha 1-3$ bonds (Figure 6), but no mannose $\alpha 1-6$ bonds were detected. These results are in agreement with the glycosyl linkages observed for other fungal and yeast proteins, usually displaying mannose $\alpha 1-2$ and $\alpha 1-3$ linkages [20]. Although mannose $\alpha 1-6$ bonds have been observed in other fungi such as Aspergillus [20], they either do not exist in $B$. cinerea, at least in the proteins analyzed, or their contribution to the proteins molecular weight is not significant.

The extracellular media obtained after growing the strains overexpressing the three glycoproteins showed an enhanced ability to elicit plant defenses, as detected in seedling growth inhibition assays (Figure 7). This was not surprising in the case of BcPG1, since this proteins has recently been shown to be recognized as a PAMP by the Arabidopsis pattern recognition receptor RBPG1 [43], but was completely unexpected for the other two. It is tempting to speculate that it is the sugar fraction on these proteins the part responsible for the elicitation of the plant defenses, since this feature is shared by the three proteins, but clearly the alternative explanation, i.e. different amino acid sequences in the three proteins act as elicitors, cannot be ruled out at this point.

\section{Conclusions}

B. cinerea secretes plenty of glycosylated proteins belonging to a diverse set of families, with polysaccharide hydrolases, proteases, and oxidoreductases being the most abundant groups. As expected, Ser/Thr-rich regions, considered to be substrates of the $O$-glycosylation machinery, are twice more abundant in the glyco-secretome than in the whole secretome. Surprisingly, proteins predicted to be hyper-O-glycosilated are more abundant in the glycosecretomes of $O$-glycosylation deficient mutants, possibly because $O$-glycosylation causes retention in the cell wall or extracellular matrix. $O$-glycosylated proteins seem to have a prominent role in plant-pathogen interaction, since the independent overexpression of three of them in $B$. cinerea increases elicitation of plant defenses by the fungus.

\section{Methods}

\section{Strains and growth conditions}

B. cinerea strains B05.10 [50] and the three bcpmt knockout mutants ( $\Delta b c p m t 1, \Delta b c p m t 2$, and $\Delta b c p m t 4)$ [28] were maintained as conidial suspensions, or as mycelium in agar plugs for strain $\Delta b c p m t 2$ (it does not produce conidia), in $15 \%$ glycerol at $-80^{\circ} \mathrm{C}$ for long storage. For routine use, fungal strains were maintained at $4^{\circ} \mathrm{C}$ in silica gel [51]. Unless otherwise indicated, B. cinerea was grown in YGG-low medium (0.5\% Yeast Extract, $10 \mathrm{mM}$ glucose, and 0.3\% Gamborg's B5), inoculated with $10^{6}$ conidia per $\mathrm{ml}$, at $20^{\circ} \mathrm{C}$ with shaking at $150 \mathrm{rpm}$. A description of all $B$. cinerea strains used in this work can be found in Table 2.

\section{Isolation and quantification of extracellular glycoproteins}

Secretome samples were obtained from fungal cultures in Petri dishes. Usually, ten plates with $20 \mathrm{ml}$ of YGGlow medium were inoculated with mycelial plugs of the indicated strains and incubated 4 days at $20^{\circ} \mathrm{C}$ in the dark, without shaking. The culture medium was then harvested by filtration through 4 layers of filter paper and frozen at $-20^{\circ} \mathrm{C}$ until use. After thawing, glycoproteins were purified by affinity chromatography on Concanavalin-A Sepharose. The culture medium (approx. $100 \mathrm{ml}$ ) was first equilibrated with $1 / 3$ volume of $4 \mathrm{X}$ binding buffer $(80 \mathrm{mM}$ Tris-HCl pH 7.4, $2 \mathrm{M} \mathrm{NaCl}, 4 \mathrm{mM} \mathrm{MnCl} 2,4 \mathrm{mM} \mathrm{CaCl} 2)$,

Table 2 B. cinerea strains used in this work

\begin{tabular}{|c|c|c|}
\hline Botrytis cinerea strain & Description & Antibiotic resistance \\
\hline B05.10 & Wild-type strain of B. cinerea [50] & None \\
\hline$\Delta B c p m t 1$ & bcpmt1 gene knock-out mutant & Hygromycin-B resistance [28] \\
\hline$\Delta B c p m t 2$ & bcpmt2 gene knock-out mutant & \\
\hline$\Delta B c p m t 4$ & bcpmt4 gene knock-out mutant & \\
\hline B05.10 (BcSpl1-GFP) & $\begin{array}{l}\text { Transformant of B05.10, expressing a fusion protein with BcSpl1 [14], } \\
\text { GFP [58], and tags (6xHis; c-myc) }\end{array}$ & \\
\hline B05.10 (BcSpl1-GFP-ST) & $\begin{array}{l}\text { Transformant of B05.10, expressing a fusion protein with BcSpl1 [14], } \\
\text { GFP [58], a Ser/Thr-rich region from Cel5A [42] and tags (6xHis; c-myc) }\end{array}$ & \\
\hline B05.10 (BCPG1) & $\begin{array}{l}\text { Transformant of B05.10, over-expressing the B. cinerea protein BcPG1, } \\
\text { with tags ( } 6 \text { xHis; c-myc) }\end{array}$ & $\begin{array}{l}\text { Nourseothricin resistance. The expression } \\
\text { construction was integrated at the niaD } \\
\text { (nitrate reductase) locus [57]. }\end{array}$ \\
\hline B05.10 (BCIEB1) & $\begin{array}{l}\text { Transformant of B05.10, over-expressing the B. cinerea protein BcIEB1, } \\
\text { with tags ( } 6 \text { xHis; c-myc) }\end{array}$ & \\
\hline B05.10 (BcSUN1) & $\begin{array}{l}\text { Transformant of B05.10, over-expressing the B. cinerea protein BCSUN1, } \\
\text { with tags (6xHis; c-myc) }\end{array}$ & \\
\hline
\end{tabular}


centrifuged (1 $000 \times \mathrm{g}, 10 \mathrm{~min})$ to remove insoluble material, and run through a Hi-Trap Con-A $4 \mathrm{~B}$ prepacked column (GE Healthcare 28-9520-85) at a flow rate of $1 \mathrm{ml} / \mathrm{min}$. Proteins binding non-specifically to the column were removed by washing with $15 \mathrm{ml}$ of $1 \mathrm{X}$ binding buffer at the same flow rate. Glycoproteins were finally eluted with elution buffer (1X binding buffer supplemented with $300 \mathrm{mM}$ methyl- $\alpha$ D-glucopyranoside), at a flow rate of $0.5 \mathrm{ml} / \mathrm{min}$, and 1-ml fractions were collected. Protein concentration was determined by the method of Bradford [52], and usually fractions 2-4 contained appreciable protein concentration and were pooled. Typically, about 25-110 $\mu \mathrm{g}$ of glycoproteins were obtained, with the higher quantity corresponding to the wild-type strain B05.10.

\section{Protein electrophoresis and identification}

Unless otherwise indicated, protein samples were concentrated for electrophoresis by precipitation with methanolchloroform [53] followed by resuspension in SDS-PAGE sample buffer. SDS-PAGEs were carried out on Any-KD Mini-PROTEAN TGX precast gels (Bio-Rad), and the gels were stained with colloidal Coomassie brilliant blue [54]. 2D electrophoresis were carried at the CNB Proteomics Facility (Centro Nacional de Biotecnología, Madrid, Spain), stained with silver, and the proteins in the indicated spots were then identified as explained elsewhere [28].

Protein identification by LC-MS/MS was carried out at the proteomics facility of the Centro de Biología Molecular Severo Ochoa (Madrid, Spain). Samples of glycoproteins were precipitated with methanol-chloroform, dried, and the pellet sent to the facility to be redissolved in SDS-PAGE sample buffer and analyzed by LC-MS/MS, basically as reported by Clemente et al. [55], but using a different database, uniprot-fungi.fasta, for peptide identification. False discovery rate was lower than 0.01 .

\section{Bioinformatics}

Signal peptide detection was performed using the SignalP 4.1 server $[34,35]$. The prediction of nonclasical protein secretion was done with the SecretomeP 2.0 server [36,37], using default parameters. Family assignment was done using sequence similarities to proteins of known function detected by BLAST $[38,56]$, and the presence of conserved domains reported by Pfam $[39,40]$. Those proteins with no high similarity to proteins with known function were labelled as dubious, but the BLAST best hit, or the Pfam family and/or conserved domains, are also reported. In these cases, the proteins are included in the corresponding family, as defined by BLAST or Pfam. Only the proteins without any clear BLAST similarity or conserved domains are included in the "Unknown" family.
In order to search for Ser/Thr-rich regions in proteins, as well as regions predicted to be hyper-O-glycosylated, we used the Microsoft Excel macro XRR previously described [21]. Positions of Ser/Thr residues along every protein, and/or positions of predicted $O$-glycosylation sites identified by NetOGlyc 4.0 [32], were transferred to an Excel spreadsheet and loaded into the XRR macro. The XRR macro basically reports a list of regions with a length of at least of 20 amino acids displaying the indicated minimum content of Ser + Thr (or predicted $O$-glycosylation sites). In order to plot the distribution of the Ser/Thr-rich regions along proteins, the central positions of all Ser/Thr-rich regions was calculated as percentage distance from the $\mathrm{N}$-terminus and grouped in ten categories $(0-10 \%, 10-20 \%, \ldots)$, as described before [21].

\section{Expression of recombinant proteins in $B$. cinerea}

PCR amplifications were made with Phusion High-Fidelity DNA Polymerase (New England Biolabs) when the DNA product was to be used in cloning experiments, and Taq polymerase (GenScript) was used in any other case. All oligonucleotides used (Additional file 2) were from Life Technologies. Yeast recombinational cloning (YRC) was carried out as described by Schumacher [57], and plasmid extraction from Saccharomyces cerevisiae was made with the E.Z.N.A. Yeast Plasmid Kit (OMEGA bio-tek).

A fusion protein composed of GFP, the $B$. cinerea cerato-platanin BcSpl1, and a Ser/Thr-rich region from the $B$. cinerea protein Cel5A (a putative substrate for $O$-glycosylation) [42], was expressed in $B$. cinerea by transformation with plasmid pNDN-GFP-ST (see below). A control protein lacking the Cel5A Ser/Thr-rich region was expressed with plasmid pNDN-GFP. Plasmid pNDNGFP was constructed by YRC introducing in plasmid pNDN-OGG [57], linearized with NcoI, a DNA fragment amplified from plasmid pCRP-GFP [41] with primer pair pCRPOGG-FW/pCRPOGG-RV and displaying i) the complete ORF of the $b c s p l 1$ gene [14], ii) the c-myc and $6 \mathrm{xHis}$ epitopes (originally from pPICZ $\alpha A$; Invitrogen, Carlsbad, CA, USA), and iii) a codon-optimized GFP gene, adapted for $B$. cinerea and originally coming from plasmid pOptGFP [58]. To obtain the pNDN-GFP-ST plasmid, a 299-bp DNA fragment containing the Ser/Thr-rich region from the endo-ß-1,4-glucanase Cel5A [42] was amplified with the primer pair pCRPOGGST-FW/ pCRPOGGST-RV, using B. cinerea B05.10 genomic DNA as template, and cloned into NotI-linearized pNDNGFP by YRC.

Three B. cinerea proteins, BcPG1 (acc. no. B0510_5388) [17], BcIEB1 (acc. no. B0510_2361) and BcSUN1 (acc. no. B0510_8844) were overexpressed in B. cinerea B05.10, fused to various epitopes, by transformation with plasmids pCBN-EPG, pCBN-IGE and pCBN-SUN, respectively, 
which were constructed as follows. An intermediate plasmid, pCBN, was first assembled by fusing with YRC the following three fragments: i) a 6583-bp fragment amplified from plasmid pBHT2 [59] with the primer pair CAMBIA-FW/CAMBIA-RV, ii) a 3452-bp fragment amplified from plasmid pNDN-OGG [57] with the primer pair NDN-CAMBIA-FW/NDN-CAMBIA-RV and digested with EcoRI and HindIII, and iii) a 4175-bp fragment also amplified from pNDN-OGG but with primer pair NDNFW/NDN-RV. In a second step, two inserts were cloned simultaneously by YCR in pCBN previously digested with NcoI plus HindIII: i) a 121-bp fragment coding for the 6xHis and c-myc eptitopes amplified from pPICZ $\alpha \mathrm{A}$ with primer pair CMYC-FW/CMYC-RV, and ii) one of the three genes coding $B$. cinerea proteins. The latter were obtained by PCR, using $B$. cinerea B05.10 genomic DNA as template and the following primer pairs: EPG1-FW/EPG1-CMICRV for pCBN-EPG (expression of BcPG1), IGE-FW/IGECMIC-RV for pCBN-IGE (expression of BcIEB1), and SUN-FW/SUN-CMIC-RV for pCBN-SUN (expression of BCSUN1).

B. cinerea transformations were carried out as described by Hamada et al. [60], with the modifications introduced by van Kan et al. [61]. The five plasmids (pNDN-GFP, pNDN-GFP-ST, pCBN-EPG, pCBN-IGE and pCBN-SUN) were transformed into $B$. cinerea by integration in the niaD locus [57]. The following fragments from the plasmids were used in the transformations: SacI-ApaI fragment from pNDN-GFP (5605 bp) or pNDN-GFP-ST (5842 bp), NotI-PstI fragment from pCBN-EPG (7398 bp) or pCBN-IGE (6868 bp), and NotI-XbaI fragment from pCBN-SUN (6898 bp). These DNA fragments were all purified from agarose gels after digestion.

Transformants were analyzed by PCRs to check for the right integration events at the niaD locus, with primer pairs binding to the transforming DNA, on one side, and to a niaD region not included in the transforming DNA, on the other (see Additional file 2 for details). Homokaryosis was confirmed in every transformant by ensuring the absence of the wild-type niaD gene with PCR (see Additional file 2). All transformants used in the rest of the work gave the expected results in these PCRs (not shown).

\section{Detection and quantification of recombinant proteins}

The secretomes of the Botrytis transformants were analyzed by SDS-PAGE and western-blots using nitrocellulose membranes (Whatman Protran BA 85) and monoclonal anti-GFP or anti-c-myc primary antibodies (Roche) diluted 1:1000. Secondary antibodies consisted of goat anti-mouse IgG conjugated to Horseradish peroxidase (Sigma-Aldrich) and were used at a 1:3000 dilution. The peroxidase was detected with Immobilon Western Chemiluminescent HRP Substrate (Millipore). Quantification of western-blot bands was done with the software Quantity One (Bio-Rad) on the chemiluminescence signal recorded with a Gel Doc XR + system (Bio-Rad).

\section{Seedling growth inhibition experiments}

Samples to be tested in these assays consisted in culture media obtained after growing $B$. cinerea for 16 hours in YGG-low medium, inoculated with $3 \cdot 10^{6}$ conidia $/ \mathrm{ml}$. These media were filtered with 4 layers of filter paper and frozen until use. Thawed culture media were first centrifuged (20 min, $16000 \mathrm{xg}$ ) and $200 \mu \mathrm{l}$ were then added into 96-well microtiter plates and incubated with Nicotinana tabacum cv. Havana seedlings. After 6-8 days in a phytotron, plants were photographed and weighted. To obtain the plants, seeds were sterilized with chlorine gas [62] and germinated in Petri dishes with $20 \mathrm{ml}$ of half-strength Murashige-Skoog medium (Pronadisa, Spain) for 20-30 days.

\section{Exo-mannosidase treatment}

Recombinant exo-mannosidases, $\alpha 1-6$ mannosidase (P0727S) and $\alpha 1-2,3$ mannosidase (P0729S), were from New England Biolabs (Ipswich, Massachusetts). Proteins in $1 \mathrm{ml}$ of culture medium were precipitated with methanol-chloroform [53], resuspended in water, and incubated with 40 units of $\alpha 1-2,3$ and/or 32 units of a1-6 mannosidases under standard conditions recommended by the supplier. Reactions were first incubated for 18 hours at $37^{\circ} \mathrm{C}$, then the same amounts of enzyme/s were added and incubation was prolonged for 2 more hours. Reactions were stopped by the addition of SDS-PAGE sample buffer.

\section{Additional files}

Additional file 1: Protein content of the Botrytis cinerea glyco-secretome.

Additional file 2: Oligonucleotides used in this study.

\section{Competing interests}

The authors declare that they have no competing interests.

\section{Authors' contributions}

NB and CG conceived the study. All authors participated in the design of the experiments as well as the analysis/evaluation of the results. MG drafted the initial manuscript and all authors participated in the editing and approved its final version.

\section{Acknowledgments}

We are grateful to Julia Schumacher for sending the vectors for site-directed integration in the B. cinerea niaD locus, and for the protocols and advice regarding YCR. Support for this research was provided by grants from the Ministerio de Educación y Ciencia (AGL2010-22222) and Gobierno de Canarias (PI2007/009). M.G. was supported by Gobierno de Canarias.

Received: 1 July 2014 Accepted: 24 September 2014

Published online: 12 October 2014 


\section{References}

1. Dean R, van Kan JA, Pretorius ZA, Hammond-Kosack KE, Di Pietro A, Spanu PD, Rudd JJ, Dickman M, Kahmann R, Ellis J, Foster GD: The Top 10 fungal pathogens in molecular plant pathology. Mol Plant Pathol 2012, 13:414-430.

2. Elad Y, Williamson B, Tudzynski P, Delen N: Botrytis spp. and diseases they cause in agricultural systems - An introduction. In Botrytis: Biology, Pathology and Control. Edited by Elad Y, Williamson B, Tudzynski P, Delen N. Dordrecht/Boston/London: Kluwer Academic Publishers; 2004:1-8.

3. Amselem J, Cuomo CA, van Kan JAL, Viaud M, Benito EP, Couloux A, Coutinho PM, de Vries RP, Dyer PS, Fillinger S, Fournier E, Gout L, Hahn M, Kohn L, Lapalu N, Plummer KM, Pradier JM, Quévillon E, Sharon A, Simon A, ten Have A, Tudzynski B, Tudzynski P, Wincker P, Andrew M, Anthouard V ̃̃, Beever RE, Beffa R, Benoit I, Bouzid O, et al:: Genomic analysis of the necrotrophic fungal pathogens Sclerotinia sclerotiorum and Botrytis cinerea. PLoS Genet 2011, 7:e1002230.

4. Staats M, van Kan JAL: Genome update of Botrytis cinerea strains B05.10 and T4. Eukaryot Cell 2012, 11:1413-1414.

5. Girard V, Cherrad S, Dieryckx C, Gonçalves I, Dupuy JW, Bonneu M, Rascle C, Job C, Job D, Vacher S: Proteomic analysis of proteins secreted by Botrytis cinerea in response to heavy metal toxicity. Metallomics 2012, 4:835-846.

6. Li B, Wang W, Zong Y, Qin G, Tian S: Exploring pathogenic mechanisms of Botrytis cinerea secretome under different ambient $\mathrm{pH}$ based on comparative proteomic analysis. J Proteome Res 2012, 11:4249-4260.

7. Espino JJ, Gutiérrez-Sánchez G, Brito N, Shah P, Orlando R, González C: The Botrytis cinerea early secretome. Proteomics 2010, 10:3020-3034.

8. Fernández-Acero FJ, Colby T, Harzen A, Carbú M, Wieneke U, Cantoral JM, Schimdt J: 2-DE proteomic approach to the Botrytis cinerea secretome induced with different carbon sources and plant-based elicitors. Proteomics 2010, 10:2270-2280.

9. Shah P, Gutiérrez-Sánchez G, Orlando R, Bergmann C: A proteomic study of pectin-degrading enzymes secreted by Botrytis cinerea grown in liquid culture. Proteomics 2009, 9:3126-3135.

10. Shah P, Atwood JA, Orlando R, El MH, Podila GK, Davis MR: Comparative proteomic analysis of Botrytis cinerea secretome. J Proteome Res 2009, 8:1123-1130.

11. González-Fernández R, Aloria K, Valero-Galván J, Redondo I, Arizmendi JM, Jorrín-Novo JV: Proteomic analysis of mycelium and secretome of different Botrytis cinerea wild-type strains. J Proteomics 2014, 97:195-221.

12. Noda J, Brito N, González C: The Botrytis cinerea xylanase Xyn11A contributes to virulence with its necrotizing activity, not with its catalytic activity. BMC Plant Biol 2010, 10:38.

13. Cuesta Arenas $Y$, Kalkman ERIC, Schouten A, Dieho M, Vredenbregt $P$, Uwumukiza B, Osés Ruiz M, van Kan JAL: Functional analysis and mode of action of phytotoxic Nep1-like proteins of Botrytis cinerea. Physiol Mol Plant Pathol 2010, 74:376-386.

14. Frías M, González C, Brito N: BcSpl1, a cerato-platanin family protein, contributes to Botrytis cinerea virulence and elicits the hypersensitive response in the host. New Phytol 2011, 192:483-495.

15. Brito N, Espino JJ, González C: The endo-ß-1,4-xylanase Xyn11A is required for virulence in Botrytis cinerea. Mol Plant Microbe Interact 2006, 19:25-32.

16. Kars I, Krooshof GH, Wagemakers $L$, Joosten R, Benen JAE, van Kan JAL: Necrotizing activity of five Botrytis cinerea endopolygalacturonases produced in Pichia pastoris. Plant J 2005, 43:213-225.

17. ten Have A, Mulder W, Visser J, van Kan JA: The endopolygalacturonase gene $B c p g 1$ is required for full virulence of Botrytis cinerea. Mol Plant Microbe Interact 1998, 11:1009-1016.

18. Valette-Collet $\mathrm{O}$, Cimerman A, Reignault P, Levis C, Boccara M: Disruption of Botrytis cinerea pectin methylesterase gene $B c p m e 1$ reduces virulence on several host plants. Mol Plant Microbe Interact 2003, 16:360-367.

19. Nafisi M, Stranne M, Zhang L, van Kan J, Sakuragi Y: The endo-arabinanase BcAra1 is a novel host-specific virulence factor of the necrotic fungal phytopathogen Botrytis cinerea. Mol Plant Microbe Interact 2014, 27:781-792.

20. Goto M: Protein O-glycosylation in fungi: diverse structures and multiple functions. Biosci Biotechnol Biochem 2007, 71:1415-1427.

21. González M, Brito N, González C: High abundance of Serine/Threonine-rich regions predicted to be hyper-O-glycosylated in the extracellular proteins coded by eight fungal genomes. BMC Microbiol 2012, 12:213.

22. Hutzler J, Schmid M, Bernard T, Henrissat B, Strahl S: Membrane association is a determinant for substrate recognition by PMT4 protein O-mannosyltransferases. Proc Natl Acad Sci U S A 2007, 104:7827-7832.
23. Fernández-Álvarez A, Marín-Menguiano M, Lanver D, Jiménez-Martín A, Elías-Villalobos A, Pérez-Pulido AJ, Kahmann R, Ibeas Jl: Identification of O-mannosylated virulence factors in Ustilago maydis. PLoS Pathog 2012, 8:e1002563.

24. Lommel M, Strahl S: Protein O-mannosylation: conserved from bacteria to humans. Glycobiology 2009, 19:816-828

25. Loibl M, Wunderle L, Hutzler J, Schulz BL, Aebi M, Strahl S: Protein O-mannosyltransferases associate with the translocon to modify translocating polypeptide chains. J Biol Chem 2014, 289:8599-8611.

26. Girrbach V, Zeller T, Priesmeier M, Strahl-Bolsinger S: Structure-function analysis of the dolichyl phosphate-mannose: protein O-mannosyltransferase ScPmt1p. J Biol Chem 2000, 275:19288-19296.

27. Willer T, Amselgruber W, Deutzmann R, Strahl S: Characterization of POMT2, a novel member of the PMT protein O-mannosyltransferase family specifically localized to the acrosome of mammalian spermatids. Glycobiology 2002, 12:771-783.

28. González M, Brito N, Frías M, González C: Botrytis cinerea protein Omannosyltransferases play critical roles in morphogenesis, growth, and virulence. PLOS ONE 2013, 8:e65924.

29. Strahl-Bolsinger S, Scheinost A: Transmembrane topology of Pmt1p, a member of an evolutionarily conserved family of protein O-mannosyltransferases. J Biol Chem 1999, 274:9068-9075.

30. ten Have A, Espino JJ, Dekkers E, Sluyter SCV, Brito N, Kay J, González C, van Kan JA: The Botrytis cinerea aspartic proteinase family. Fungal Genet Biol 2010, 47:53-65.

31. NetNGlyc 1.0. In [http://www.cbs.dtu.dk/services/NetNGlyc]

32. NetOGlyc 4.0. In [http://www.cbs.dtu.dk/services/NetOGlyc]

33. Steentoft C, Vakhrushev SY, Joshi HJ, Kong Y, Vester-Christensen MB, Schjoldager KT, Lavrsen K, Dabelsteen S, Pedersen NB, Marcos-Silva L, Gupta R, Bennett EP, Mandel U, Brunak S, Wandall HH, Levery SB, Clausen H: Precision mapping of the human O-GalNAc glycoproteome through SimpleCell technology. EMBO J 2013, 32:1478-1488.

34. SignalP 4.1. In [http://www.cbs.dtu.dk/services/SignalP].

35. Petersen TN, Brunak S, von Heijne G, Nielsen H: SignalP 4.0: discriminating signal peptides from transmembrane regions. Nature Methods 2011, 8:785-786

36. SecretomeP 2.0. In [http://www.cbs.dtu.dk/services/SecretomeP].

37. Bendtsen JD, Jensen LJ, Blom N, von Heijne G, Brunak S: Feature-based prediction of non-classical and leaderless protein secretion. Protein Eng Des Sel 2004, 17:349-356.

38. BLAST. In [http://blast.ncbi.n/m.nih.gov/Blast.cgi].

39. Finn RD, Mistry J, Tate J, Coggill P, Heger A, Pollington JE, Gavin OL, Gunasekaran P, Ceric G, Forslund K, Holm L, Sonnhammer ELL, Eddy SR, Bateman A: The Pfam protein families database. Nucl Acids Res 2010, 38:D211-D222

40. Pfam. In [http://pfam.xfam.org/].

41. Frías M, Brito N, González M, González C: The phytotoxic activity of the cerato-platanin BcSpl1 resides in a two-peptide motif in the protein surface. Mol Plant Pathol 2014, 15:342-351.

42. Espino JJ, Brito N, Noda J, González C: Botrytis cinerea endo-ß-1,4-glucanase Cel5A is expressed during infection but is not required for pathogenesis. Physiol Mol Plant Pathol 2005, 66:213-221.

43. Zhang L, Kars I, Essenstam B, Liebrand TW, Wagemakers L, Elberse J, Tagkalaki P, Tjoitang D, van den Ackerveken G, van Kan JA: Fungal endopolygalacturonases are recognized as Microbe-Associated Molecular Patterns by the Arabidopsis receptor-like protein RESPONSIVENESS TO BOTRYTIS POLYGALACTURONASES 1. Plant Physiol 2014, 164:352-364.

44. Gómez-Gómez L, Felix G, Boller T: A single locus determines sensitivity to bacterial flagellin in Arabidopsis thaliana. Plant J 1999, 18:277-284

45. Gómez-Gómez L, Boller T: FLS2: an LRR receptor-like kinase involved in the perception of the bacterial elicitor flagellin in Arabidopsis. Mol Cell 2000, 5:1003-1011.

46. Pfund C, Tans-Kersten J, Dunning FM, Alonso JM, Ecker JR, Allen C, Bent AF: Flagellin is not a major defense elicitor in Ralstonia solanacearum cells or extracts applied to Arabidopsis thaliana. Mol Plant Microbe Interact 2004, 17:696-706.

47. Fernández-Álvarez A, Elías-Villalobos A, Ibeas Jl: The O-mannosyltransferase PMT4 is essential for normal appressorium formation and penetration in Ustilago maydis. Plant Cell 2009, 21:3397-3412. 
48. Girrbach $V$, Strahl S: Members of the evolutionarily conserved PMT family of protein O-mannosyltransferases form distinct protein complexes among themselves. J Biol Chem 2003, 278:12554-12562.

49. Kriangkripipat T, Momany M: Aspergillus nidulans Pmts form heterodimers in all pairwise combinations. FEBS Open Bio 2014, 4:335-341.

50. Büttner $P$, Koch F, Voigt $K$, Quidde $T$, Risch $S$, Blaich R, Brückner B, Tudzynski $P$ : Variations in ploidy among isolates of Botrytis cinerea: implications for genetic and molecular analyses. Curr Genet 1994, 25:445-450.

51. Delcan J, Moyano C, Raposo R, Melgarejo P: Storage of Botrytis cinerea using different methods. J Plant Pathol 2002, 84:3-9.

52. Bradford MM: A rapid and sensitive method for the quantitation of microgram quantities of protein utilizing the principle of protein-dye binding. Anal Biochem 1976, 72:248-254.

53. Wessel D, Flügge UI: A method for the quantitative recovery of protein in dilute solution in the presence of detergents and lipids. Anal Biochem 1984, 138:141-143.

54. Neuhoff $V$, Arold N, Taube D, Ehrhardt W: Improved staining of proteins in polyacrylamide gels including isoelectric focusing gels with clear background at nanogram sensitivity using Coomassie Brilliant Blue G-250 and R-250. Electrophoresis 1988, 9:255-262.

55. Clemente P, Peralta S, Cruz-Bermudez A, Echevarría L, Fontanesi F, Barrientos A Fernandez-Moreno MA, Garesse R: hCOA3 stabilizes cytochrome c oxidase 1 (COX1) and promotes cytochrome coxidase assembly in human mitochondria. J Biol Chem 2013, 288:8321-8331.

56. Altschul SF, Gish W, Miller W, Myers EW, Lipman DJ: Basic local alignment search tool. J Mol Biol 1990, 215:403-410.

57. Schumacher J: Tools for Botrytis cinerea: New expression vectors make the gray mold fungus more accessible to cell biology approaches. Fungal Genet Biol 2012, 49:483-497.

58. Leroch M, Mernke D, Koppenhoefer D, Schneider P, Mosbach A, Doehlemann G, Hahn M: Living colors in the Gray Mold pathogen Botrytis cinerea: Codon-optimized genes encoding green fluorescent protein and mCherry, which exhibit bright fluorescence. Appl Environ Microbiol 2011, 77:2887-2897.

59. Mullins ED, Chen X, Romaine P, Raina R, Geiser DM, Kang S: Agrobacteriummediated transformation of Fusarium oxysporum: an efficient tool for insertional mutagenesis and gene transfer. Phytopathology 2001, 91:173-180

60. Hamada W, Reignault P, Bompeix G, Boccara M: Transformation of Botrytis cinerea with the hygromycin B resistance gene, hph. Curr Genet 1994, 26:251-255.

61. van Kan JAL, van't Klooster JW, Wagemakers CAM, Dees DCT, van der Vlugt-Bergmans CJB: Cutinase A of Botrytis cinerea is expressed, but not essential, during penetration of gerbera and tomato. Mol Plant Microbe Interact 1997, 10:30-38.

62. Clough SJ, Bent AF: Floral dip: a simplified method for Agrobacterium-mediated transformation of Arabidopsis thaliana. Plant J 1998, 16:735-743.

\section{Submit your next manuscript to BioMed Central and take full advantage of:}

- Convenient online submission

- Thorough peer review

- No space constraints or color figure charges

- Immediate publication on acceptance

- Inclusion in PubMed, CAS, Scopus and Google Scholar

- Research which is freely available for redistribution 